\title{
DEL HOMBRE-MÁQUINA A LA MÁQUINA-HOMBRE: \\ Materialismo, mecanicismo y transhumanismo
}

\author{
Martín López Corredoira \\ Instituto de Astrofísica de Canarias, \\ La Laguna (Tenerife)
}

Resumen: El materialismo de la Edad Moderna nos describe al hombre como una máquina, comparable a un complejo artilugio mecánico. Cabe entonces imaginar que una máquina no-biológica pueda constituir un ser pensante como lo son los seres humanos, e incluso cabría pensar en la posibilidad de codificación de una mente humana real para su posterior trasvase a un sustrato artificial. Considero que estas últimas posiciones son más propias de la cultura friki o de amantes de la ciencia ficción que de una cultura humanista seria. En cualquier caso, una cosa parece clara: la simulación por ordenador de cualquier tipo de materia no es la materia misma. Una simulación por ordenador de una estrella no emite luz ni calor, y del mismo modo tampoco ofrece calor humano (en términos materiales) ni voluntad de vivir una máquina que hipotéticamente pueda contener toda la información sobre el ser humano y simular sus respuestas.

Palabras clave: materialismo, mecanicismo, transhumanismo, transferencia mental, voluntad de vivir.

\section{From man-machine to machine-man: materialism, mechanicism and transhumanism}

Abstract: The materialism of the Modern Age describes human beings as machines, comparable to complex mechanical devices. It is then possible to imagine that a non-biological machine can constitute a thinking being as humans are, and one could even think of the possibility of coding a real human mind for its subsequent transfer to an artificial substrate. I think that these latter positions are more typical of the geek culture or of science fiction lovers than of a serious humanist culture. In any case, one thing seems clear: the computer simulation of any type of matter is not the matter itself. A computer simulation of a star does not emit light or heat, and likewise a machine that hypothetically can contain all the information about a human being and simulate his/her responses offers neither human heat (in material terms) nor will to live. 
Keywords: materialism, mechanicism, transhumanism, mind uploading, will to live.

Recibido: 17/08/2018 Aprobado: 20/02/2019

\section{Materialismo y mecanicismo de los médicos-filósofos en los siglos XVI-XIX}

El materialismo es una concepción del mundo muy antigua. Cierto es que ha habido cambios a lo largo de los siglos a la hora de determinar qué es la materia, pero no así en su posición más básica que se traduce en un "todo es materia". En lo que toca al tema de la mente humana, el "no hay mente sino cerebro" viene de hace unos dos mil quinientos años con Hipócrates, o quizás antes. "Los hombres deberían saber que del cerebro, y nada más que del cerebro, vienen las alegrías, el placer, la risa y el ocio, las penas, el dolor, el abatimiento y las lamentaciones" — decía Hipócrates. Esta afirmación podría también salir de la boca de un materialista de la Edad Moderna o Contemporánea.

Hay una tradición mecanicista dentro de los médicos-filósofos (o filósofos-médicos) de entre los siglos XVI y XIX, pensadores que ejercieron su condición de médicos profesionalmente, o al menos tuvieron un contacto bastante cercano con la Medicina, y coligieron de sus estudios que la mecánica del funcionamiento del cuerpo de animales o humanos se asemeja a la del resto de la Naturaleza.

El médico y filósofo español Antonio Gómez Pereira (1500-ca. 1560) fue un pionero en estas ideas mecanicistas en los seres vivos, que defendía en su visión del "automatismo de las bestias". Para él, “...la naturaleza ha creado a los irracionales tan semejantes a los humanos que, con las mismas afecciones por las que nosotros nos sentimos menos bien, los brutos también se mueven peor e irracionalmente" (Antoniana Margarita). Gómez Pereira no era, sin embargo, un materialista porque guardaba para el ser humano una concepción fuera de la materia. Su materialismo se restringió al mundo animal no-humano. Es ésta una visión casi idéntica a la del filósofo francés René Descartes (1596-1650) casi un siglo después, quien argumentaría que los animales no humanos son máquinas, pura materia sometida a 
leyes de la Naturaleza y carentes de pensamiento, mientras que los seres humanos tienen materia y mente pensante (dualismo). René Descartes no fue médico de profesión, pero sí frecuentaba las facultades de Medicina, presenciando disecciones, y aportando incluso alguna disquisición en su obra escrita sobre cuestiones de anatomía y fisiología.

Un paso más allá da el materialista francés Julien Offray de La Mettrie (1709-1751), quien no distinguiría entre hombres y animales y afirmaría que todos ellos son máquinas. Si Gómez Pereira o Descartes afirmaban que los animales eran máquinas y los hombres poseían una naturaleza dual de materia y espíritu, La Mettrie simplifica el esquema eliminando la clase especial de seres humanos y dándoles a éstos la categoría de máquinas, es decir materia sometida a leyes mecánicas. Observa este pensador y conocedor del cuerpo humano a través de su oficio de médico que la misma necesidad que acontece a los fenómenos naturales sucede en el cuerpo humano y, asimismo, en los contenidos del llamado "espíritu" o "alma". La misma rigidez mecánica presente en cualquier artilugio mecánico compuesto de engranajes, resortes, etc., está presente en el ser humano. Una naturaleza material, y por ende determinista como predecían las leyes de Newton para toda la materia, es la que caracteriza todo acontecer en el sistema mecánico "ser humano". La Mettrie afirma:

...si lo que se piensa en mi cerebro no es una parte de esta víscera y, por consiguiente, de todo el cuerpo, ¿̇por qué cuando, tranquilo en mi cama, trazo el plan de una obra o sigo un razonamiento abstracto, mi sangre se calienta? [...] Porque, en fin, si la tensión de los nervios, que constituye el dolor, causa la fiebre por la cual el espíritu se turba y no tiene ya voluntad, y si, recíprocamente, el espíritu que trabaja demasiado perturba el cuerpo y enciende ese fuego de consunción que se llevó a Bayle en una edad tan poco avanzada, si tal titilación me hace querer, me fuerza a desear ardientemente aquello de lo cual no me preocupaba en absoluto un momento antes, si a su vez ciertos rastros en el cerebro provocan el mismo prurito y los mismos deseos, ¿́por qué duplicar lo que es evidentemente uno? (El hombre máquina) 
También fue médico y materialista el pensador alemán Friedrich Karl Christian Ludwig Büchner (1824-1899), quien en su obra Fueræa y materia afirma:

Todo cuanto hemos dicho al hablar de las relaciones de la fuerza y de la materia, nos conduce a afirmar que las leyes naturales y racionales son siempre idénticas. Lo que llamamos espíritu, entendimiento, inteligencia, se compone de fuerzas naturales, aunque combinadas de una manera particular, que por su parte, y como cualquier otra fuerza natural, sólo puede manifestarse en ciertas y determinadas materias. Hallándose éstas combinadas en la vida orgánica de un modo indefinidamente complicado y bajo formas particulares, producen efectos que nos parecen a primera vista maravillosos e inexplicables, mientras que los procedimientos y efectos todos del mundo inorgánico son infinitamente más sencillos, y por consiguiente más fáciles de comprender. En el fondo, sin embargo, es siempre la misma materia, y la experiencia nos enseña a cada paso que las leyes de la inteligencia son las leyes del mundo.

\section{2. ¿Piensan los ordenadores?}

Siguiendo la lógica precedente, si los hombres son máquinas que piensan por procesos materiales y mecánicos existentes en sus cerebros, el paso siguiente es preguntarse: ¿no será posible hacer una máquina no-biológica lo suficientemente sofisticada para competir en complejidad con el cerebro humano y no podrían esas máquinas pensar como lo hacen los seres humanos?

Existen numerosos trabajos en esta línea de pensamiento dedicados a la comparación de mentes y ordenadores (Jackendoff, 1987; Johnson-Laird, 1988). Con la aparición de los modelos computacionales de la mente surge en la década de 1950 la corriente del computacionalismo. Según ésta, la mente funciona como un ordenador de algún tipo. Nuestro "Yo" es el análogo al producto de ciertas computaciones más que a las computaciones en sí, y el cerebro sería la máquina computadora. El computacionalismo clásico utiliza el modelo de computador clásico (digital, simbólico, con una estructura de 
von Neumann, etcétera). Un período más moderno corresponde al conexionismo, en el que también se compara el cerebro humano con un computador, pero más complejo (analógico, no-simbólico, con estructura de red neuronal, etc.). El computacionalismo o el conexionismo nos dicen que la mente funciona como un ordenador de algún tipo.

Sin embargo, en general, se puede decir que ni siquiera las redes neuronales más complejas alcanzan a emular las conexiones neuronales en el cerebro (Crick, 1994: cap. 13). Las redes neuronales pueden dar alguna explicación a ciertas funciones del cerebro: memoria, pensamiento exacto, distinción y abstracción de los componentes esenciales de los patrones en los estímulos, conducta con propósitos aparentes, conducta adaptativa, etc., pero no son cerebros humanos en cuanto a funcionamiento lógico. El cerebro guarda parte de la memoria en los mismos lugares donde produce operaciones comunes (Crick, 1994: cap. 13). Software y hardware, distinguibles en un ordenador, están completamente entremezclados en el cerebro. Además, el cerebro se desarrolla a sí mismo durante el crecimiento y aun en la edad adulta, reemplazando conexiones y generando nuevas neuronas (Kempermann et al., 1997; Koch y Laurent, 1999).

En mi opinión, las máquinas que denominamos inteligencia artificial no han logrado emular la vida, ni hace 50 años, ni en el presente, ni tampoco me parece que el desarrollo tecnológico nos lleve a tales logros ni en otros 50 años. Se presume, sí, de tener artilugios autómatas que reproducen con gran exactitud la conducta de algunos animales, pero falta lo fundamental en ellos para ser comparables a los seres vivos: la voluntad de vivir. Las máquinas construidas por el hombre no tienen deseos, no tienen apetitos ni temores, sólo computan y aprenden dentro de esquemas lógicos, pero carecen del elemento más característico: las pulsiones irracionales.

\section{Transhumanismo}

La corriente de pensamiento del transhumanismo se propuso como lema transformar la condición humana mediante el desarrollo y fabricación de 
tecnología posiblemente disponibles en un futuro (cambios en el ADN, prótesis,...), que mejoren las capacidades humanas, tanto a nivel físico como psicológico o intelectual. Aquí se da otro salto cualitativo en la empresa de aunar los seres biológicos pensantes con las máquinas no-biológicas. Se apuesta por la construcción de un nuevo tipo de sujetos (u objetos, según se mire): una combinación de elementos vivos naturales o artificialmente modificados con componentes robóticas y electrónicas, unos individuos mixtos fusión de seres humanos naturales con modificaciones genéticas e implantes artificiales.

La idea puede resultar cercana en cierta medida si uno se restringe a las posibilidades más simples: incluso ya antes del siglo XX existían los implantes como en el caso de piezas rígidas para substituir algunas partes óseas del cuerpo. La típica imagen del pirata con la pata de palo podría ser un transhumanismo de muy bajo nivel, o los implantes dentales para substituir la dentición original. Algo más sofisticados son ya los aparatos para sordos, o los marcapasos para el corazón. No obstante, todo esto se reduce a posibilidades que no modifican las capacidades psicológicas, cognitivas, intelectuales, no modifican el carácter o las capacidades mentales de los seres humanos, al menos no directamente. Pero el transhumanismo no se queda en meros implantes corporales, sino que se plantea modificaciones del ser humano que afectan a su sistema nervioso, y aquí es donde toca el problema filosófico de la identidad humana y hasta qué punto se puede seguir considerando humano a un híbrido de máquina y hombre.

La primera mención de la palabra transhumanismo la dio Julien Huxley (1887-1975) en un artículo homónimo del año 1957. Julien fue un biólogo, escritor y humanista británico, hermano del escritor Aldous Huxley y nieto del biólogo gran defensor del darwinismo Thomas Henry Huxley. Quizá no sea una casualidad que publicara su artículo en la década del descubrimiento de la estructura de la molécula de ADN y las posibilidades de ingeniería genética que ahí se vislumbraban, pero no menciona tal futurible en su artículo. Como quiera que sea, el pensamiento transhumanista no 
tendría apenas impacto en la década de 1950 y no sería hasta los años 70 y más aún en los años 80 cuando se convertiría en una tendencia intelectual.

Fereidoun M. Esfandiary (1930-2000), quién firma sus obras como FM2030, fue un filósofo y futurólogo iraní que publicaría en 1989 una obra con título Are You a Transhuman?, que alcanzaría cierto impacto y estimularía la corriente en cuestión. Que un "pitoniso" con un pseudónimo que parece una emisora de radio sea uno de los máximos representantes de esta tradición de pensamiento en la actualidad ya nos dice bastante sobre qué tipo de movimiento se trata: cultura friki para amantes de la ciencia ficción y las pseudociencias.

Uno de los últimos desafíos que presenta el transhumanismo es el plantearse la transferencia mental (Mind uploading, en inglés; Goertzel y Ikle, 2012; Bamford, 2012; Sotala y Valpola, 2012), que, en el contexto de la ciencia ficción, nos habla de la posibilidad futura de un hipotético proceso de codificación de una mente real para su posterior transvase a un sustrato artificial, algún tipo de máquina y ordenador. El ordenador podría entonces ejecutar un modelo que simula el procesado de información del cerebro transferido, de modo que responda igual que el original ante los mismos estímulos y sea indistinguible del mismo. Es éste precisamente el tema de la cuestión que da nombre a nuestro curso de verano en Ávila.

\section{Consideraciones}

Pienso que hay dos tipos de consideraciones importantes a realizar ante el desafío que da título a este curso de verano: "¿Es posible transferir la mente humana a un soporte no biológico?”.

En primer lugar, que este tipo de discusiones es más propio de otro tipo de ámbitos que el llamado filosófico. El contexto y los orígenes del problema provienen del ámbito de la literatura fantástica y de la ciencia-ficción y en tal debieran de quedarse, no como una cosa seria a abordar por intelectuales, sino como un mero entretenimiento de niños, adolescentes y algunos adultos con mentalidad más juvenil. A los clientes de este tipo de 
planteamientos cabe más bien englobarlos dentro de la llamada cultura friki (palabra que proviene del inglés freak, que significa algo así como monstruo, raro, estrafalario, extravagante), de monstruos de la tecnología, nada a tomar demasiado en serio por quienes se preocupan por la cultura humanista seria. Cabe englobarlo en la tradición de experimentos literarios como el de la obra que este año cumple 200 años: Frankenstein, de Mary Shelley. Las mismas fantasías de literatura de ciencia-ficción de hace dos siglos nos llegan hoy en día con un nuevo ropaje, y de hecho la propuesta sigue siendo igualmente monstruosa, espeluznante: hacer un ser humano a base de juntar trozos de distinta procedencia, sólo que en el caso del Frankenstein de Shelley se trataba de juntar distintos componentes orgánicos de distintos seres humanos y en la moderna propuesta transhumanista se aboga por mezclar partes biológicas y partes no-biológicas, o incluso puramente no-biológicas aportando el ser humano sólo la información contenida en su cerebro. Entretenido, sí, pero los filósofos se preocupan por cómo son o cómo debieran ser las cosas, y no es de mayor interés desgranar los entresijos de la literatura mitológica con seres inventados, ni hablar de centauros, mitad hombres y mitad caballos, ni de videojuegos. De hecho, hasta donde yo sé, la famoso obra de Shelley no ha suscitado debates filosóficos relevantes en su época hace dos siglos, había temas más importantes para los importantes filósofos de la primera mitad del s. XIX que considerar las historias de monstruitos ficticios. Si hoy las cosas son diferentes, no es mérito de la actual literatura fantástica con respecto a la obra de Shelley, sino demérito de la actual filosofía. Es preocupante, sí. Y es que si dejamos que la actual generación de cultura friki o el hombre-masa protecnológico invada las humanidades terminaremos enviándonos whatsapps en vez de escribir y leer elaborados libros y artículos, o aprobando las buenas ideas dándoles un "me gusta" en Facebook, o poniendo minimensajes en Twitter en vez de escribir reseñas, y terminaremos substituyendo en los programas de estudio el vitalismo de Nietzsche por una pseudofilosofía del tipo "que la fuerza te acompañe" de la saga de Star Wars. 
Dentro del presente curso de verano en Ávila, José Manuel Elena Ortega nos ha increpado numerosas veces a prestar atención al vertiginoso avance de la robótica y la inteligencia artificial en los últimos años, de los que cada poco se publican infinidad de avances, los cuales nosotros, pobres filósofos ignorantes de la sabiduría de nuestros tiempos, no logramos entender por estar desfasados tal cual abuelo que va a la zaga con respecto a las juventudes actuales en el uso de teléfonos móviles y ordenadores. Parece insinuarse que debieran dejarse de lado a los grandes pensadores clásicos que se han preocupado por entender la vida y la inteligencia y debiera ponerse más atención y esfuerzo en leer alguno de esos gruesos volúmenes acerca de la tecnología digital en cada versión modificada que sale cada poco tiempo. Pienso sin embargo que nada nuevo hay bajo el sol de la computación: los mismos conceptos que se aprendían en los años 50 o 60 son lo que perviven hoy en día; sólo la creciente miniaturización y los consiguientes incrementos de capacidad de almacenamiento de datos y de velocidad de los procesos de cálculo han cambiado, pero tales son cambios cuantitativos, no cualitativos. Por otra parte, dejar que ingenieros informáticos o electrónicos guíen el camino del intelecto sobre nuestra comprensión de la inteligencia y la vida me parece tan demencial como dejar que un zapatero dé lecciones a un caminante viajero sobre cómo es el mundo. Obviamente, con unos buenos zapatos caminamos mejor y podemos recorrer más mundo, y con unos buenos ordenadores facilitamos muchas labores científicas. Pero ahí termina la labor del artesano, y un ingeniero no es más que un artesano, no ningún intelectual; poco cabe aprender de tal en cuestiones espirituales o intelectuales. Lamentablemente, el mundo actual está viviendo una nueva era dentro de su actual decadencia cultural: la era tecnocrática. Si Auguste Comte dividía en tres etapas la Historia de la Humanidad dominadas respectivamente por el sacerdote, el filósofo metafísico y el científico positivista, hoy nuestros artesanos fabricantes de máquinas computadoras y robotitos quieren ser los nuevos referentes fundamentales de toda actividad intelectual humana, por encima del orden espiritual, filosófico y científico. Así nos va... y así ocurre que en nuestra ciencia cada vez se respira menos 
inteligencia y sí más embrutecimiento tecnológico. Y así ocurre que, quienes poseen una visión deformada del mundo pensando que todo se reduce a colecciones de bits con valores de 0 o 1 y que tanto distan de la comprensión de lo que es la vida, quieren dar lecciones al mundo sobre tal y ofrecer espejismos o delirios de grandeza, alimentados por infantiles sueños de literatura fantástica, jugando a ser pequeños dioses, ante la idea de que está en manos de tal gremio la creación de la vida y la inteligencia.

Con todo, el filósofo también puede hacer uso de ejemplos literarios o de experimentos mentales (Gedankenexperiment, en alemán) irrealizables en la práctica, para ejercitar su mente o tratar de entender conceptos más mundanos que el de la ficción utilizada. Y si se trata de poner a prueba nuestro resbaladizo concepto de consciencia, puede tener su utilidad. Al fin y al cabo, como dijera Borges, la metafísica es una rama de la literatura fantástica que no busca la verdad ni la verosimilitud, sino el mero asombro, y en ese sentido sí cabe ver una cierta proximidad entre la cultura friki y la metafísica, como la había en su tiempo entre la religión y la metafísica.

La segunda consideración que quiero realizar es que, si hemos de entrar a tomar cierta seriedad en el asunto, lo más importante a remarcar es que simular algo con un ordenador no es lo mismo que aquello que se simula. $\mathrm{Y}$ aunque fuese posible tecnológicamente copiar toda la información del cerebro y reproducir en una máquina los procesos neuronales (lo que no creo que vayamos a ver en nuestras vidas, ni nuestros nietos tampoco), el ser humano es algo más que un conjunto de datos, con lo cual nunca podremos encerrar lo esencial del ser humano en una computadora u otros soportes no biológicos.

Es una trivialidad esto que vengo apuntando, pero es que a veces la solución a arduas cuestiones filosóficas viene dada por respuestas elementales antes de que algunas mentes gusten de enredar y hacer el problema más complicado de lo que parece. La cosa es sencilla: como Spinoza decía, "el concepto de perro no ladra”. No estoy abogando aquí por un alma o una parte inmaterial irremplazable en la esencia del ser humano, no, eso se lo 
dejo a mis colegas religiosos. Es más bien que, desde el propio planteamiento materialista, la materia y sus estructuras son algo más que un conjunto de datos almacenables en la memoria de un ordenador. La materia es materia y no meramente la descripción de la misma.

Yo trabajo como astrofísico y conozco las simulaciones que se realizan para emular estrellas y galaxias y la formación de estructuras a gran escala del Universo. Funcionan muy bien, y prácticamente cualquiera de las características que se reproducen de los astros ajustan los datos que se observan con telescopios de sus equivalentes reales. Ahora bien, una simulación hecha por ordenador de una estrella no da calor ni luz como la auténtica, tan sólo nos da unos números que representan su luminosidad en distintas frecuencias. Del mismo modo, una simulación de un ser humano, por muy exacta que fuera, no es un ser humano, no da calor "humano", no tiene sangre caliente, no tiene vísceras, no tiene hormonas interactuando con el cerebro, no tiene toda la eclosión de fenómenos químicos que se dan en nuestros cuerpos y que una máquina no-biológica puede calcular pero no hacer real lo que calcula. El fenómeno biológico que constituyen nuestros cuerpos es materia, sí, pero materia insustituible por algo que no sea su igual. Simular no es igualar. El sueño de los materialistas de reducir el hombre a materia ha triunfado, pero hacer de la materia inerte hombres no es posible. Se pueden construir engendros mecánicos, sí, y es posible que los límites de la robótica y la electrónica nos lleven a máquinas cuasiantropomórficas, pero nunca serán hombres, no pueden serlo, no comen, no defecan, no orinan, no duermen, no respiran, no sudan, no maman, no eyaculan o menstrúan, no lloran verdaderas lágrimas, no tienen todo el conjunto de realidades que tiene el ser humano. Y, como he señalado anteriormente, las máquinas no tienen voluntad de vivir, y una simulación de un ser humano tampoco la tendrá porque la simulación de un deseo no es el deseo mismo, ni la simulación de sus miedos es el miedo mismo. El hombre o cualquier ser vivo sobre la Tierra es un ser orgánico, visceral, voluntarioso, apegado a la química del carbono. Cualquier imitación es un mero juguete. 
Martín López Corredoira

\section{Bibliografía}

S. Bamford, "A framework for approaches to transfer of a mind's substrate", en International Journal of Machine Conciousness, 2012 (4(1)), pp. 23-34.

L. Büchner, Kraft und Stoff (1854). Traducido al español en: Fueræa y materia. Estudios populares de historia y filosofía naturales, Valencia, F. Sempere y Comp, 1905. Reproducido en: Oviedo, Fundación Gustavo Bueno, 1999.

F. Crick, The Astonishing Hypothesis: The Scientic Search for the Soul, Londres, Simon and Schuster, 1994. Traducido al español en: La búsqueda científica del alma, Madrid, Debate, 1994.

F. M. Esfandiary, Are You a Transhuman?: Monitoring and Stimulating Your Personal Rate of Growth in a Rapidly Changing World, New York, Warner Books, 1989.

B. Goertzel, M. Ikle, "Special issue on mind uploading. Introduction", en International Journal of Machine Conciousness, 2012 (4(1)), pp. 1-3.

A. Gómez Pereira, Antoniana Margarita (1554), Medina del Campo. Traducido al español en: Antoniana Margarita, Oviedo, Fundación Gustavo Bueno, Oviedo, 2000.

J. Huxley, "Transhumanism", en New Bottles for New Wine, London, Chatto \& Windus, 1957, págs. 13-17.

R. Jackendoff, Conciusness and the Computational Mind, Cambridge (Massachussets, Estados Unidos), Bradford Books, MIT Press, 1987.

P. N. Johnson-Laird, Computer and the Mind: An Introduction to Cognitive Science, Cambridge (Massachussets, Estados Unidos), Harvard University Press, 1988.

G. Kempermann, H. G. Kuhn, F. H. Gage, "More hippocampal neurons in adult mice living in an enriched environment”, Nature, 1997 (386), p. 493.

C. Koch, G. Laurent, "Complexity and the Nervous System”, Science, 1999 (284), pp. 96-98.

J. O. de La Mettrie, L'Homme Machine (1749, Leiden). Traducido al español en: El hombre máquina, Buenos Aires, Universitaria de Buenos Aires, 1961.

M. Shelley, Frankenstein, or the modern Prometheus, London, Lackington, Hughes, Harding, Mavor \& Jones, 1818.

K. Sotala, H. Valpola, "Coalescing Minds: Brain Uploading-Related Group Mind Scenarios, en International Journal of Machine Conciousness, 2012 (4(1)), pp. 293-312.

Martín López Corredoira

fuego.templado@gmail.com 